BNL-107664-2015-JA

\title{
Hierarchical, Ultrathin Single-Crystal Nanowires of CdS Conveniently Produced in Laser-Induced Thermal Field
}

\author{
Li-Li Han ${ }^{\dagger}$, , Huolin L. Xin ${ }^{\ddagger}$, Sergei A. Kulinich*, ${ }^{\S}, "$, Li-Jun Yang ${ }^{\dagger}$, Xi-Wen Du*,† \\ ${ }^{\dagger}$ School of Materials Science and Engineering, Tianjin University, Tianjin 300072, China \\ ${ }^{\ddagger}$ Center for Functional Nanomaterials, Brookhaven National Laboratory, New York 11973, USA \\ ${ }^{\S}$ Institute of Innovative Science and Technology, Tokai University, Hiratsuka, Kanagawa 259-1292, Japan \\ "Aston Institute of Photonic Technologies, Aston University, Birmingham, B4 7ET, UK
}

\begin{abstract}
Hierarchical nanowires (HNWs) exhibit unique properties and have wide applications, while often suffering from imperfect structure. Herein, we report a facile strategy toward ultrathin CdS HNWs with monocrystal structure, where a continuous-wave (CW) Nd:YAG laser is employed to irradiate an oleic acid (OA) solution containing precursors and a light absorber. The high heating rate and large temperature gradient generated by the CW laser lead to the rapid formation of tiny zinc-blende CdS nanocrystals which then line up into nanowires with the help of OA molecules. Next, the nanowires experience a phase transformation from zinc-blende to wurtzite structure, and the transformation-induced stress creates terraces on their surface, which promotes the growth of side branches and eventually results in monocrystal HNWs with an ultrathin diameter of $24 \mathrm{~nm}$. The one-step synthesis of HNWs is conducted in air and completes in just 40 seconds, thus being very simple and rapid. The prepared CdS HNWs display photocatalytic performance superior to their nanoparticle counterparts, thus showing promise for catalytic applications in

the

future.
\end{abstract}

\section{INTRODUCTION}

Hierarchical nanowires (HNWs) with specific properties, such as structural hierarchy, large surface area and direct electron transport pathways, ${ }^{1}$ have been considered as ideal candidates for diverse applications in energy conversion and storage, ranging from solar cells, ${ }^{2,3}$ photocatalysis ${ }^{4,5}$ and water splitting, ${ }^{6}$ to $\mathrm{Li}$-ion batteries, ${ }^{7}$ supercapacitors, ${ }^{8,9}$ and light emitting diodes..$^{10}$ So far, various methodologies and approaches have been developed for the synthesis of such HNWs, including sequential catalyst-assisted vapor-liquid-solid (VLS) ${ }^{11,12}$ or solution-liquid-solid approaches, ${ }^{13}$ solution growth on pre-formed nanowires (NWs), ${ }^{14-16}$ screw-dislocation in combination with VLS, ${ }^{17}$ one-step self-catalytic growth, ${ }^{18,19}$ and oriented attachment of branched nanoparticles. ${ }^{20}$ As a result, various HNWs, such as brush-like $\mathrm{SnO}_{2} / \mathrm{ZnO}$ heterobranched structures, ${ }^{21}$ brush-like $\mathrm{ZnO},{ }^{19}$ tree-like $\mathrm{GaP}^{12}$ and comb-like $\mathrm{ZnS}^{22}$ homobranched structures have been obtained. However, the existing approaches are typically nontrivial in realization, time-consuming, and require air-free conditions, ${ }^{23}$ while their products often exhibit poor crystallinity, ${ }^{1}$ backbone growth along polar direction, ${ }^{24}$ and low branching density. ${ }^{25}$ In addition, the obtained HNWs are usually very thick, with their width over 1 $\mu \mathrm{m}$, though ultrathin branched NWs have a larger surface area, faster charge transport, and better water wettability. ${ }^{26,27}$ Therefore, the direct preparation of high-quality ultrathin HNWs via a simple route still remains a big challenge.

Compared to common heating sources, the continuous-wave (CW) laser beam can generate extremely high temperatures for the synthesis of nanostructures. For example, a Nd:YAG 1064-nm CW laser with high power was already employed successfully to prepare carbon nanotubes. ${ }^{29,30}$ Especially, laser beam can heat liquid phase locally to a high temperature and produce a gradient temperature field along radial directions. ${ }^{28}$ High temperature accelerates reactions and thus leads to supersaturated regions formed in the beam center. ${ }^{31}$ Violent convection induced by the gradient field transports the supersaturated solution into lower-temperature areas, leading to a burst of nucleation and formation of nanostructures, in accordance with the Wilson-Frenkel growththeory. ${ }^{28}$

In view of the above, we adopted a near-infrared CW laser to irradiate a precursor solution (with a specially added dye) for the synthesis of ultrathin CdS HNWs. Specifically,N,N,N,N-tetrakis(4-dibutylaminophenyl)-p-benzoquinonebis(iminiumhexafluoroantimonate) was chosen as a light absorber, cadmium acetate and sulfur powder as $\mathrm{Cd}$ and $\mathrm{S}$ precursors, and oleic acid (OA) as a solvent. Upon irradiating the mixture for merely a few tens of seconds, a large amount of HNWs were obtained with their backbone along the $\left[1 \overline{2}^{\overline{2}} \mathrm{o}\right]$ direction and sidearms along the $\left\langle 1^{\overline{1}}{ }_{\mathrm{OO}}>\right.$ and $\left[{ }^{1}{ }^{\overline{1}}\right]$ directions. It is worth noting that the novel approach is low-cost, simple and time-efficient. The CdS structures 
prepared via laser heating exhibited higher photocatalytic efficiency in decaying $\mathrm{RhB}$ when compared to $\mathrm{CdS}$ nanoparticles prepared via conventional heating method. This study thus demonstrates a new, simple and efficient approach for the synthesis of nanomaterials with complex morphology.

\section{EXPERIMENTAL SECTION}

Synthesis of CdS hierarchical nanowires via continuous-wave laser irradiation. $0.2665 \mathrm{~g}$ of $\mathrm{Cd}(\mathrm{Ac})_{2} \cdot 3 \mathrm{H}_{2} \mathrm{O}$ and $0.032 \mathrm{~g}$ of S powder were added into $20 \mathrm{~mL}$ of $\mathrm{OA}$ and magnetically stirred at $100{ }^{\circ} \mathrm{C}$ for $15 \mathrm{~min}$ to form 0.05 M OA solution. $0.036 \mathrm{~g}$ of dye, N,N,N',N',-tetrakis(4-dibutylaminophenyl)-p-benzoquinonebis(iminium hexafluoroantimonate), was ultrasonically dispersed in $20 \mathrm{~mL}$ of OA. $0.4 \mathrm{~mL}$ of the dye in OA $(1.8 \mathrm{~g} / \mathrm{L})$ was mixed with $0.2 \mathrm{~mL}$ of $0.05 \mathrm{M} \mathrm{Cd}(\mathrm{Ac})_{2}$ and $0.2 \mathrm{~mL}$ of $0.05 \mathrm{M}$ sulfur solutions in a quartz tube with a diameter of $1 \mathrm{~cm}$. The $\mathrm{CW}$ Nd:YAG laser (500 W average power) beam was focused on the center of the prepared liquid mixture. The laser spot size and power density were adjusted to $0.8 \mathrm{~mm}$ and $100 \mathrm{~kW} / \mathrm{cm}^{2}$, respectively. After laser irradiation for $40 \mathrm{os}$, the tube was cooled to ambient temperature and the product was extracted for characterization.

Synthesis of CdS nanoparticles via conventional heating. The reaction conditions were the same as those used in the above described laser-assisted synthesis of CdS HNWs, except that an external heat source, furnace (set as $250^{\circ} \mathrm{C}$ ), was used instead of laser. The temperature inside the reaction mixture was in-situ monitored by a temperature sensing probe. After every $10 \mathrm{~s}, 200 \mu \mathrm{L}$ of the mixture were taken from reaction system for characterization.

Photocatalytic degradation of RhB. $15 \mathrm{mg}$ of CdS HNWs (as catalyst) were added to aqueous RhB solution (0.02 $\mathrm{mol} / \mathrm{L}, 50 \mathrm{~mL}$ ), magnetically stirred in the dark for $30 \mathrm{~min}$ to reach the adsorption-desorption equilibrium between $\mathrm{RhB}$ and the catalyst, and then exposed to visible light $\left(150 \mathrm{~mW} / \mathrm{cm}^{2}\right)$. A UVCUT400 filter was placed inside the vessel to preclude irradiation below $400 \mathrm{~nm}$, allowing irradiation only in the visible-light wavelength range for the $\mathrm{RhB} / \mathrm{CdS}$ system. For comparison, the photocatalytic activity of the CdS nanoparticles with an average size of $10 \mathrm{~nm}$ and commercial P25 $\left(\mathrm{TiO}_{2}\right)$ powder were also tested under the same experimental conditions. UV-vis absorption spectra were recorded with 10-min intervals by using a UV-vis-infrared spectrophotometer to monitor the catalytic reaction.

Tomographic reconstruction. The annular dark-field scanning transmission electron microscopy (ADF-STEM) tilt series was acquired from -60 to $64^{\circ}$ at two-degree intervals in an instrument emitting at $200 \mathrm{keV}$. No noticeable mass loss was observed during the acquisition process. The acquired tilt series was first coarsely aligned using cross correlation. The final registrations were made manually using a Matlab script package (e-Tomo) written by R. Hovden (Muller group, Cornell) with contributions from H. Xin. The 3-D dataset was reconstructed by the simultaneous iterative reconstruction algorithm implemented in Matlab. 15 iterations were used for the final refinement.

Measurements and analysis. Transmission electron microscopy (TEM) analysis was performed on an FEI Technai G2 F2O TEM with a field emission gun operating at $200 \mathrm{kV}$. The 3D structure of CdS hierarchical NWs was determined by using 3D electron tomography with high-angle annular dark-field (HAADF) scanningtransmission electron microscopy (STEM). The composition was analyzed with an Oxford INCA energy-disperse X-ray spectroscopy (EDS) module attached to the TEM. TEM samples were prepared by dropping diluted products onto carbon-coated Cu grids and rinsing with ethanol several times. X-ray diffraction (XRD) patterns were measured by a Bruke D8 advance instrument. UV-vis-infrared absorption spectra were recorded on a Hitachi U-4100 spectrophotometer. The mixture of $2 \mathrm{~mL}$ of ethanol and $0.1 \mathrm{~mL}$ of OA was used as a reference and the mixture of $2 \mathrm{~mL}$ of ethanol and $0.1 \mathrm{~mL}$ of obtained product was taken for absorption measurement. Thermograms in CdS HNW formation process were recorded by a 100HT digital infrared camera from IRON company (USA).

\section{RESULTS AND DISCUSSION}

Figures 1a,1b and Figure S1 show that the obtained product was radially branched NWs with an average lateral dimension of $24 \mathrm{~nm}$ and lengths in the range of several microns. The average thickness of backbones and branches was measured as 6 and $9 \mathrm{~nm}$, respectively, according to TEM images. Further structural analysis of the NWs was directly provided using electron tomography, where $3 \mathrm{D}$ tomograms were reconstructed based on a series of $2 \mathrm{D}$ annular darkfield STEM images recorded at different tilt angles. The 3D tomographic reconstruction shown in Figure 1c and Figure S2 visualizes the $3 \mathrm{D}$ morphology of the product. The NWs are well seen to exhibit branches spreading in three directions, discerning a hierarchical nanowire (HNW) structure. The HRTEM image in Figure $1 \mathrm{~d}$ confirms that the HNWs are single-crystalline in structure, though with some defects. The lattice spacings of $3.58 \AA$ and $2.0 \AA$ correspond to the wurtzite $\mathrm{CdS}\left(1^{\overline{1}} \mathrm{Oo}\right)$ and $\left(11^{\overline{2}} \mathrm{O}\right)$ planes, respectively. The results are further confirmed by the selected area electron diffraction (SAED) pattern (see the inset of Figure 1d), which was indexed to the reflections of hexagonal CdS crystal along the [0oo1] direction. The TEM analysis illustrates that the HNWs have their backbone axis along the [11 $\overline{2}$ o] direction and the branches along the $\left[1^{1} \bar{l}_{00},\left[\overline{1}_{100}\right]\right.$, [ooo ${ }^{\overline{1}}$ directions. However, the reflections of the $\left(11^{2}\right.$ o) planes are unusually stronger than those of the wurtzite $\left({ }_{1} \overline{1} \mathrm{OO}\right)$ and $\left({ }_{11} \overline{2} \mathrm{O}\right)$ planes, implying the presence of some zincblende phase that manifests through its (220) planes. Thus both phases appear to coexist in the HNWs. The EDS analysis of the sample gave the $\mathrm{Cd}$ to $\mathrm{S}$ atomic ratio of $\sim 1: 1$ (Figure 1e), as expected for a stoichiometric CdS crystal. 
XRD analysis (Figure 1f) also supports the coexistence of both wurtzite and zinc-blende phases, which is consistent with the result of SAED analysis.
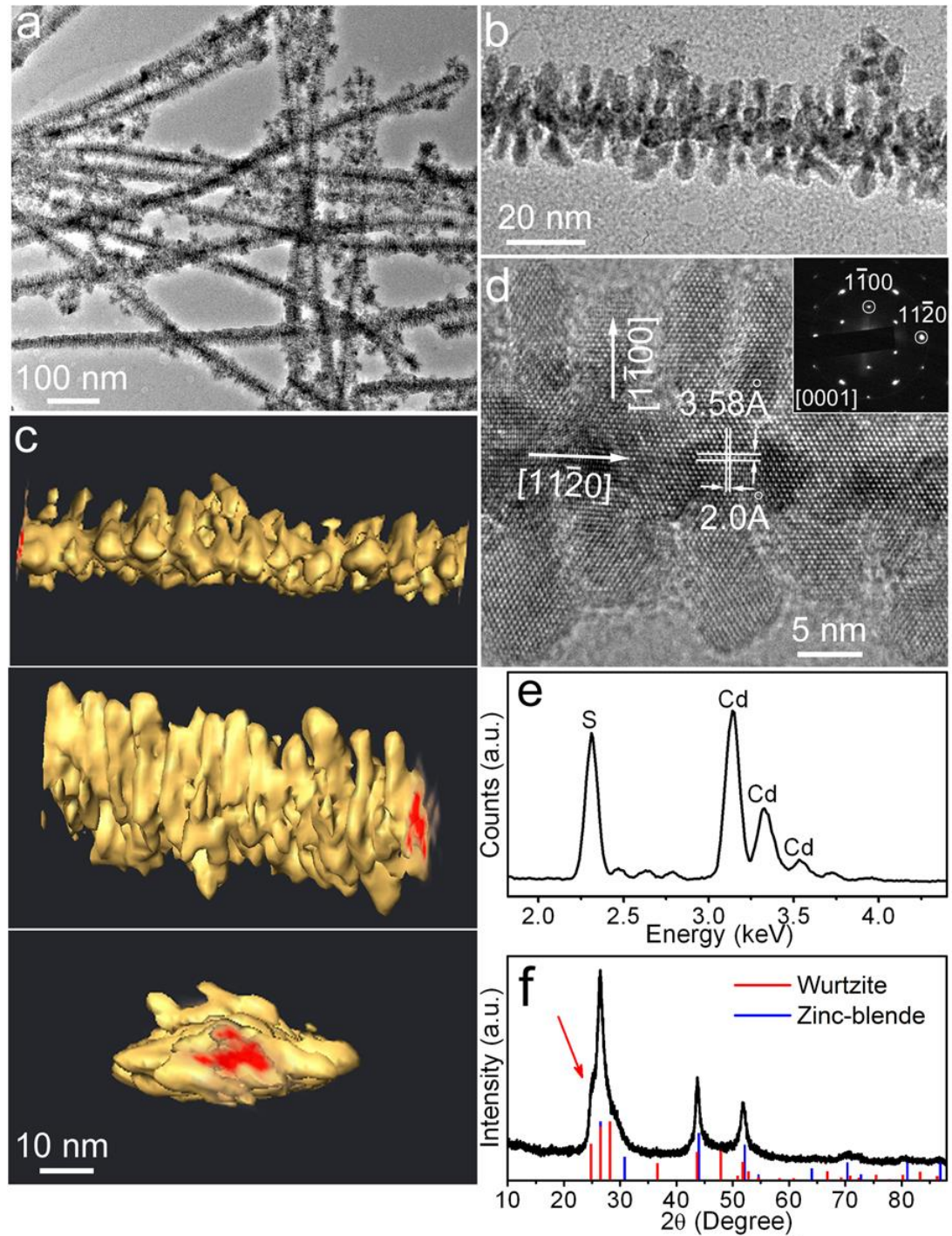

Figure 1. Characterizations of CdS HNWs prepared at $\mathrm{Cd}$ and $\mathrm{S}$ concentrations of $0.05 \mathrm{M}$ and dye concentration of $1.8 \mathrm{~g} / \mathrm{L}$ : (a) TEM image, (b) TEM image of a single NW, (c) visualization of the tomographic reconstruction of the NW from three orientations, (d) HRTEM image, (e) EDS spectrum, and (f) XRD pattern.

We further identify the influence of process conditions on the morphology of the products. First, we investigated the effect of dye concentration. At a lower concentration $(1 \mathrm{~g} / \mathrm{L})$, the product comprises mainly of irregular nanocrystals and very few NWs (see Figures S3a1-a3). At a high dye concentration $(2.6 \mathrm{~g} / \mathrm{L})$, the product consists of CdS HNWs which show thicker backbones (about $15 \mathrm{~nm}$ ) and shorter branches (about $5 \mathrm{~nm}$ ) than those obtained at the dye concentration of $1.8 \mathrm{~g} / \mathrm{L}$ (see Figures $\mathrm{S}_{3} \mathrm{~b} 1-\mathrm{b} 3$ ). These results imply that a moderate dye concentration is necessary for the formation of HNWs with desired parameters. Next, we probe the effect of precurs or concentration. At lower concentrations of Cd and S precursors (0.03 M), bare NWs instead of HNWs were produced (see Figures S4a1-S4a3). When the concentrations reached o.5 M, CdS nanotetrahedrons were produced without any HNWs (see Figures S4b1-S4b3).

To monitor temperature evolution during the process, thermograms of the beam-irradiated zone were recorded. In the absence of dye, the mixture temperature was observed to be constant over irradiation time, and so was the absorption spectrum (Figure $\mathrm{S}_{5}$ ). As the dye was added as a light absorber, the temperature increased with irradiation time and decreased radially from the laser beam center, where the temperature was recorded by a digital infrared camera placed $70 \mathrm{~cm}$ away from the reaction vessel (Figure 2a). This confirmed that the dye molecules absorbed the laser energy efficiently and heated the reaction mixture rapidly. In contrast, a common electric furnace was found to heat the mixture much slower (see Figure 2b). For instance, it only took $14 \mathrm{~s}$ for laser irradiation to reach to $150{ }^{\circ} \mathrm{C}$, while the same temperature was obtained in the furnace after $80 \mathrm{~s}$ (see Figure $2 \mathrm{~b}$ ). As a result, the product of such a 
slower heating was irregular CdS nanoparticles with the coexistence of the zinc-blende and wurtzite phases, instead of CdS HNWs (Figures S6c and S6d).
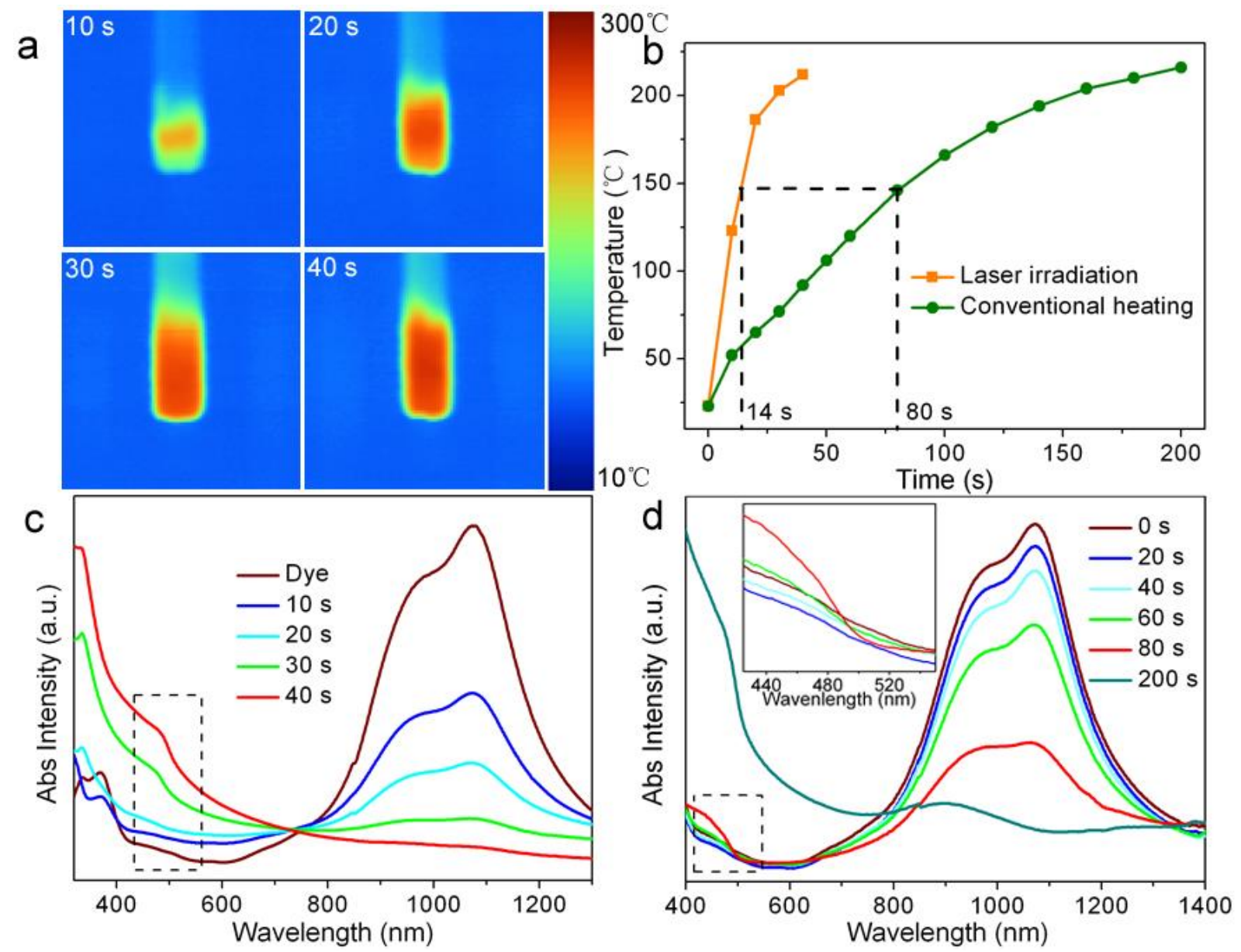

Figure 2. (a) Evolution of thermograms of the laser-irradiated reaction mixture over time measured by a digital infrared camera from the direction vertical to the laser beam. (b-d) Comparison of processes with laser irradiation and conventional heating: (b) temperature evaluation in the central zone of the mixture over time; (c,d) absorption spectra of the laserirradiated (c) and furnace-heated (d) reaction mixtures over time.

Absorption spectra of the reaction mixture were recorded to collect more information on the CdS nucleation and growth. As shown in Figure 2c, the absorption peak of organic dye at $1070 \mathrm{~nm}$ disappears gradually under laser irradiation, while that of $\mathrm{CdS}$, which is typically observed at $500 \mathrm{~nm}$, emerges after laser irradiation for $20 \mathrm{~s}$, indicating the gradual dye decomposition along with CdS formation. For comparison, in the conventionally-heated mixture of the same composition the CdS absorption peak only emerges after $80 \mathrm{~s}$ (see Figure 2d). These results clearly demonstrate that the CdS product prepared via laser irradiation has a shorter nucleation period compared to its counterpart obtained via conventional heating.

The product formed in the conventionally-heated mixture was only observed after $80 \mathrm{~s}$ and comprised of isolated CdS nanoparticles with sizes over $7 \mathrm{~nm}$ and poor crystallinity (see Figures S6a and S6b). In contrast, ultrafine nuclei $(\sim 3 \mathrm{~nm})$ formed just after $14 \mathrm{~s}$ of laser irradiation (Figure 3a), with their SAED pattern suggesting a cubic structure (see the inset of Figure 3a). This agrees well with the earlier reported finding on the zinc-blende phase being more stable in CdS nanocrystals smaller than $4 \mathrm{~nm} .{ }^{32}$ Following the nucleation, the formed nanocrystals gradually aligned and connected along a certain direction. As shown in Figure 3c, three nanocrystals with sizes around $3 \mathrm{~nm}$ lined up along the [110] direction, with their atomic model shown in Figure 3b. Later on, more nanocrystals joined the assembly, growing into a long NW with an undeveloped hierarchical structure (Figure 3d). The HRTEM image illustrates that the tip area of the NW in Figure 3d comprises of wurtzite grains which are separated by zinc-blende areas, with the wurtzite [11 $\overline{2}$ o] direction parallel to the zinc-blende [110] direction (Figures 3e and $3 \mathrm{f}$ ). Noticeably, some branches are budding from the wurtzite grains, as shown in Figures $3 \mathrm{~g}$ and $3 \mathrm{~h}$. The bottom part of the NW shown in Figure $3 \mathrm{~d}$ presents a well- 
grown area, where the zinc-blende phase disappears completely, and the branches grow epitaxially from the backbone along the $<1 \overline{1}_{\mathrm{OO}}>$ directions (Figures $3 \mathrm{i}$ and $3 \mathrm{j}$ ).

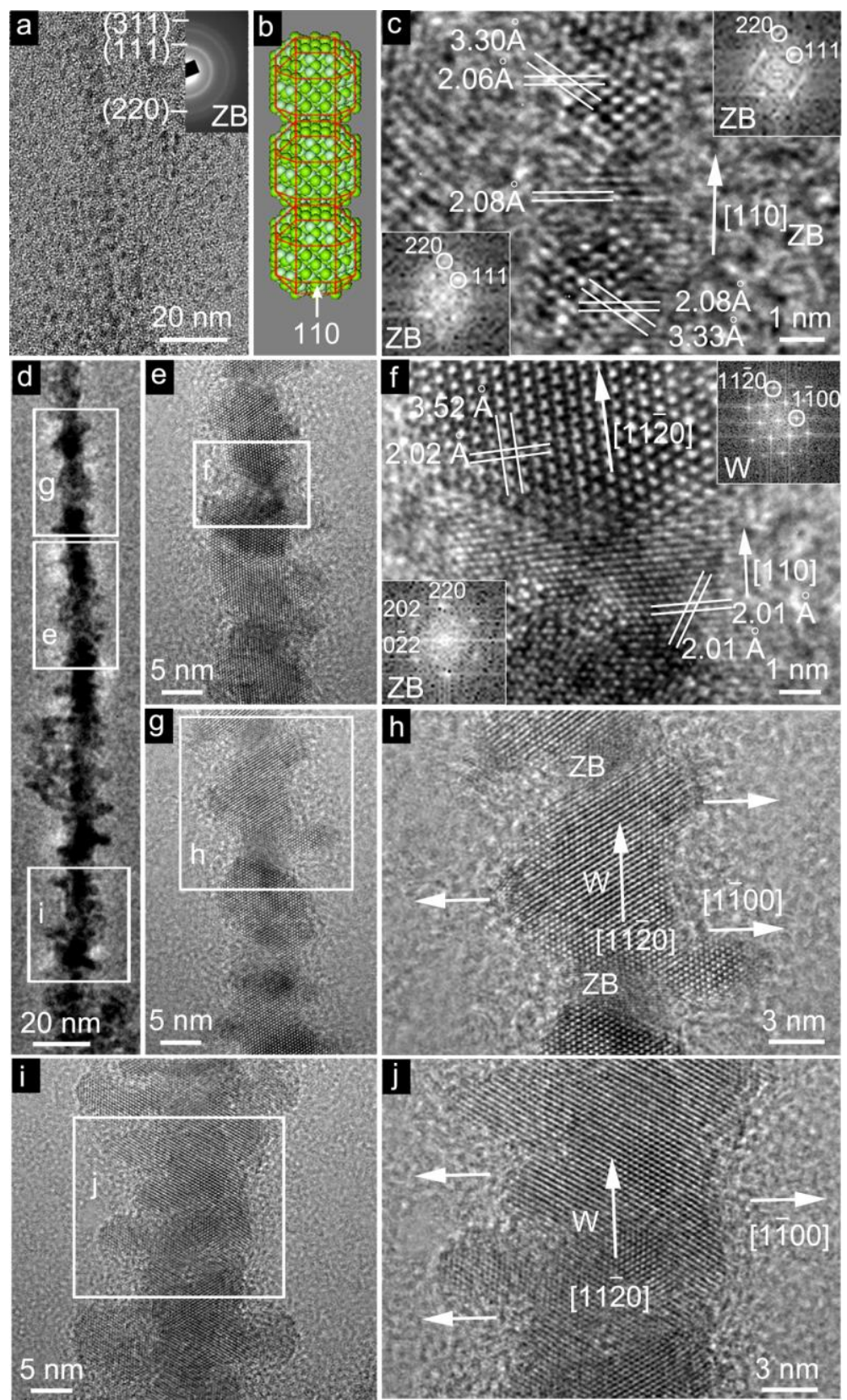

Figure 3. Characterization of CdS HNWs and their constituents: (a) TEM image; (b) atomic model and (c) HRTEM image of zinc-blende CdS nanocrystals formed after laser irradiation for $14 \mathrm{~s}$, the FFT patterns in the insets of panel (c) were indexed as the reflection of the zinc-blende structure along the $\left[1_{1}^{\overline{1}} \mathrm{o}\right.$ ] direction; (d) TEM image of one undeveloped HNW after laser irradiation for $40 \mathrm{~s}$; (e), (g) and (i) HRTEM images of different parts of the NW in panel (d). (f), (h) and (j) are magnified images of corresponding parts in panels (e), (g) and (i), where W and ZB denote wurtzite and zinc-blende phases, respectively. The FFT patterns in the top-right and bottom-left corners of panel (f) were indexed as the reflections of the wurtzite structure along the [0o01] direction and the zinc-blende one along the $\left[1^{\overline{1}}{ }_{1}\right]$ direction, respectively.

Based on the above, we propose a possible mechanism of CdS HNW formation which is schematically shown in Figure 4. The high heating rate achieved by high-energy laser irradiation (see Figure $2 b$ ) induces fast reactions and causes a high concentration of CdS nuclei in the focal area. Meanwhile, the convection arising from the temperature gradient field moves the nuclei immediately to zones with lower temperatures, leading to a significant supersaturation and thus rapid formation of CdS nanocrystals with small sizes $(\sim 3 \mathrm{~nm})$, which is in accordance with the Wilson-Frenkel 
growth theory. ${ }^{28}$ The zinc-blende phase was reported to be more stable for CdS nanocrystals smaller than 4 nm. ${ }^{22}$ Hence, in this work the tiny nanocrystals observed at the very early stage emerged as a zinc-blende phase. It was also reported that OA molecules can bind strongly with $\{001\}$ and $\{111\}$ facets of zinc-blende CdS, leaving $\{110\}$ facets exposed.33 This explains well why the tiny CdS nanocrystals connected to each other through their uncapped \{110\} facets along the [110] direction, leading to the formation of NWs (see Figure 4b2). At the same time, the coarsened grains in NWs are expected to transform gradually into the wurtzite phase as the latter is more stable at larger crystallite sizes..$^{32}$ Because the atomic structure of the zinc-blende (220) plane is similar to that of the wurtzite $\left(11^{2} \mathrm{o}\right)$ plane (see the crosssectional image in Figure $4 \mathrm{~b} 3$ ), the transformed wurtzite phase tends to grow along the $\left[1 \overline{2}^{\bar{o}} \mathrm{o}\right]$ direction. On the other hand, the lattice mismatch between the wurtzite and zinc-blende segments should cause an interface stress which can be released through the sliding of crystal planes, leading to numerous terraces on the NW surface. At this stage, newly arriving CdS species are expected to attach preferentially at terrace sites, ${ }^{34}$ resulting in the epitaxial growth of branches (Figure 4b4). The branches might grow along both the $\left\langle 1^{1} \mathrm{OO}>\right.$ and [ooo ${ }^{\overline{1}}$ ] directions. However, the [0oo1] direction is suppressed since the (0001) facets terminated with Cd atoms are efficiently capped by OA molecules. 35 Therefore, the NWs eventually grow into the HNW structure with a backbone along the $\left[{ }_{11} \overline{2} \mathrm{o}\right]$ direction and branches along the $\left[1^{\overline{1}}\right.$ oo], $\left[\overline{1}_{100}\right.$, and [ooo $\left.{ }^{\overline{1}}\right]$ directions.

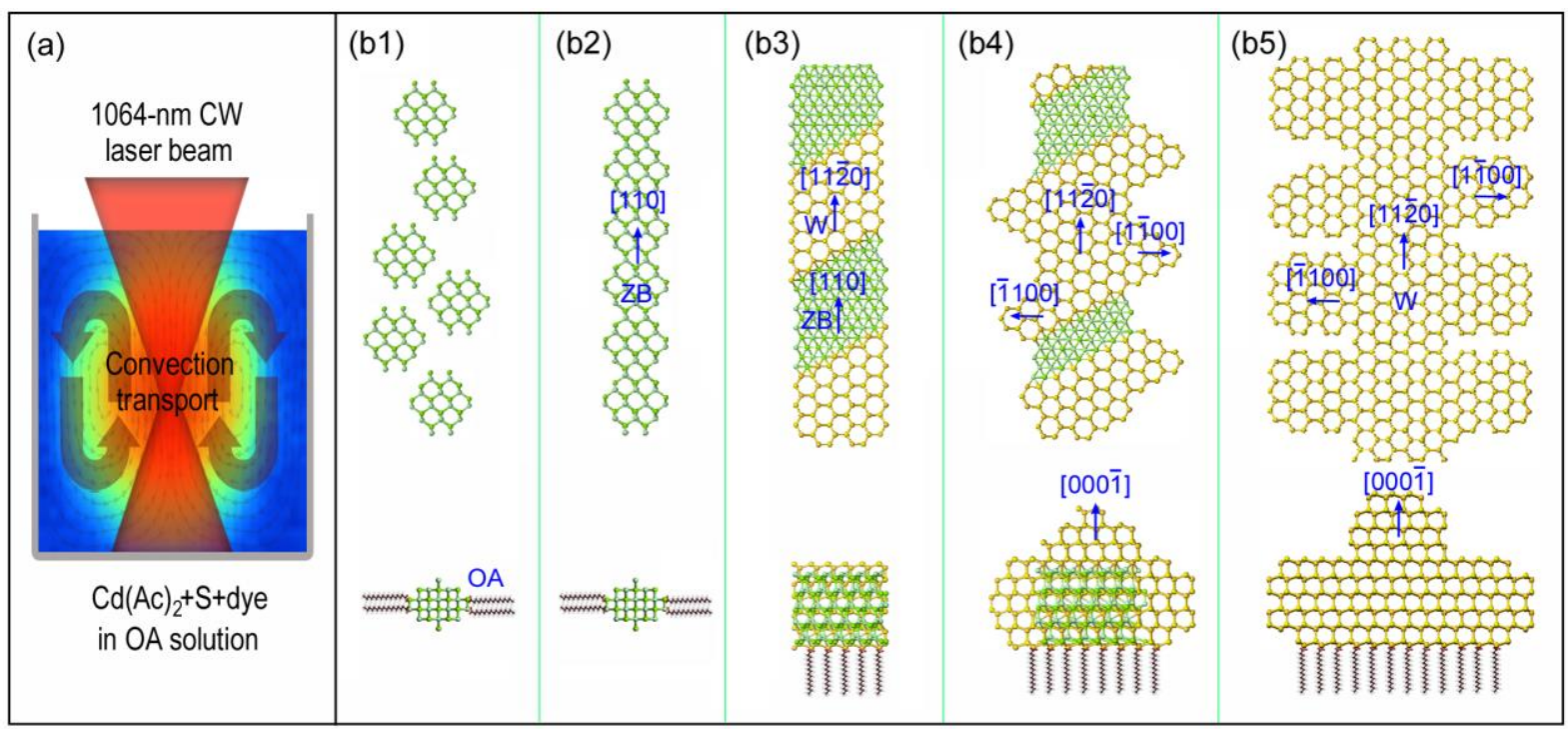

Figure 4. Proposed mechanism for the growth of HNWs: (a) simulated fluid dynamics in the vicinity of laser-heated spot, with color changes indicating temperature gradient and red and blue representing high and low temperatures, respectively; (b) 2D atomic model of HNW formation process, with top and bottom images presenting side- and cross-sectional views of a NW at different growth stages.

We tested the photocatalytic properties of the prepared CdS HNWs under visible light irradiation after successfully stripping off $\mathrm{OA}$ from their surface. The characteristic absorption at about $553 \mathrm{~nm}$ was selected to monitor the adsorption of rhodamine $\mathrm{B}(\mathrm{RhB})$ in water and its photocatalytic degradation. As shown in Figure $5 \mathrm{a}$, after irradiation for $10 \mathrm{~min}$, the absorption peak at $553 \mathrm{~nm}$ corresponding to $\mathrm{RhB}$ diminished abruptly with a concomitant wavelengthshift towards the blue region. When the illumination time was extended to $40 \mathrm{~min}$, RhB was completely decayed by the CdS HNWs. For comparison, the CdS nanoparticles synthesized via conventional heating (see Supporting Information Figures S6c and S6d for details) and a commercial Degussa P25 titania powder were selected as reference. Within the chosen period of time, the photodegradation rate in the presence of the titania nanoparticles was rather slow (Figure 5b). The photocatalytic efficiency of the CdS nanoparticles and CdS HNWs was very close, with the efficiency of the latter nanostructures being slightly higher. It should be noted that the former HNWs completely decayed RhB after only 40 min while the latter NPs decayed as much as $95 \%$ of the same dye after 70 min. This indicates that the CdS HNWs are more efficient photocatalysts than the nanoparticles. The higher photocatalytic efficiency of the CdS HNWs is probably attributed to their unique structure that facilitates faster charge transport against the recombination of excitons, which makes them more promising for photocatalytic decomposition of organic pollutants compared to CdS nanoparticles prepared via conventional heating. 

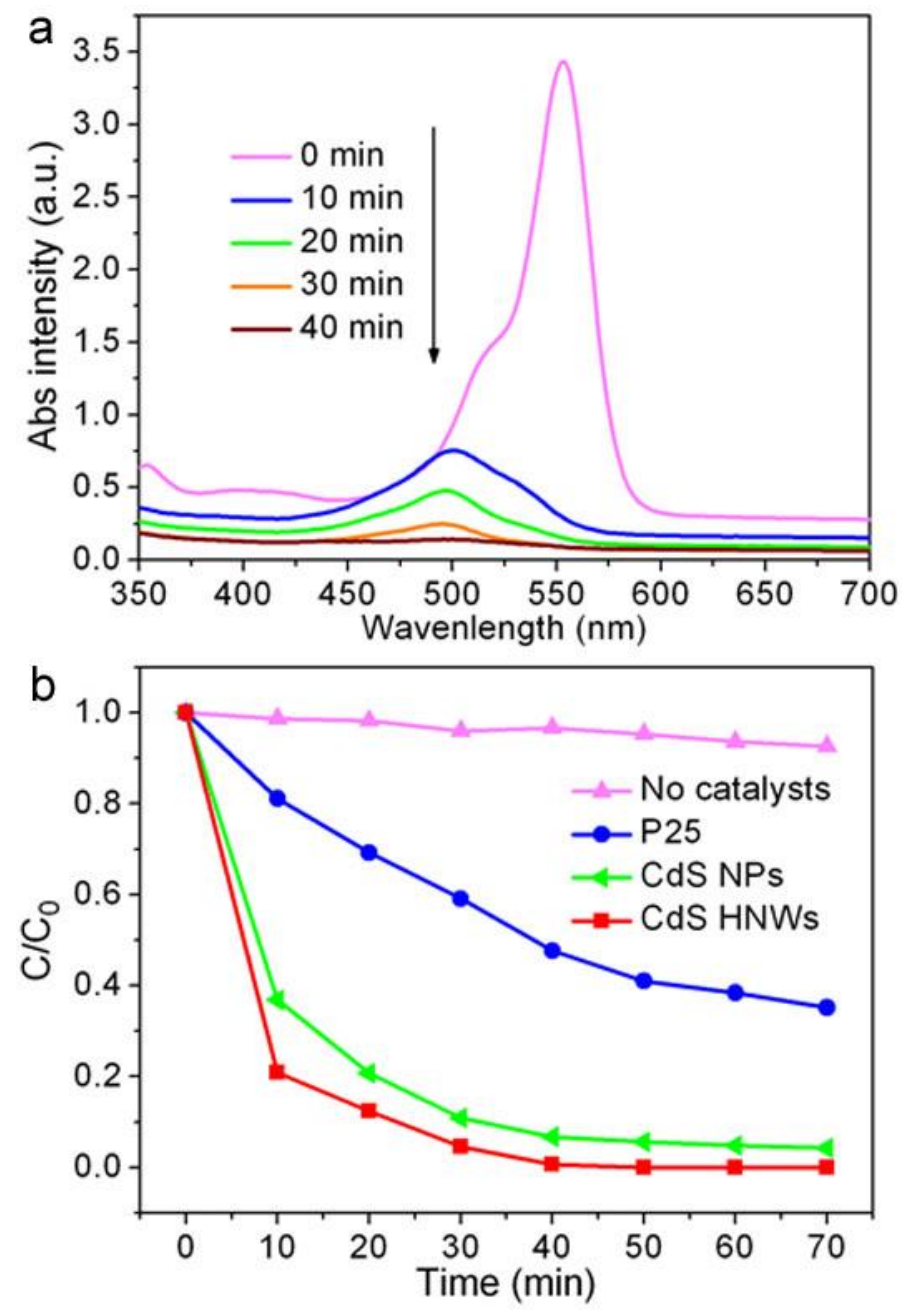

Figure 5. (a) Absorption spectra of RhB solution in the presence of CdS HNWs exposed to visible light. (b) Comparison of photocatalytic degradation of $\mathrm{RhB}(0.02 \mathrm{~mol} / \mathrm{L}, 50 \mathrm{~mL})$ in the presence of P25 titania nanoparticles, CdS nanoparticles, and CdS HNWs (15 mg of photocatalyst in each case) under visible-light irradiation.

\section{CONCLUSIONS}

We developed a new, simple and fast approach to generate a large-temperature-gradient field by irradiating a liquid reaction mixture containing precursors and a photo absorber with a continuous-wave Nd:YAG laser. This field initiates the intense nucleation and growth of tiny primary CdS nanocrystals $\sim 3 \mathrm{~nm}$ in diameter and with exposed $\{110\}$ facets. Driven by intense convection, such CdS nuclei then move to zones with lower temperatures where they assemble into nanowires through their uncapped $\{110\}$ facets. Next, the nanowires experience a phase transformation from zincblende to wurtzite and then branch growth, eventually resulting in well-developed hierarchical nanowires. The obtained CdS structures exhibit higher photocatalytic efficiency in decaying $\mathrm{RhB}$ when compared to their CdS nanoparticle counterparts prepared via conventional heating.

\section{ASSOCIATED CONTENT}

\section{Supplemental Material}

Additional TEM and STEM images of CdS HNWs, slices of three-dimensional tomographic reconstruction, CdS nanostructures obtained at different concentrations of dye, $\mathrm{Cd}$ and $\mathrm{S}$ precursors, $\mathrm{CdS}$ nanoparticles synthesized via external heating by a conventional furnace, Abs spectra of a reaction mixture of $\mathrm{Cd}(\mathrm{Ac})_{2}$ and $\mathrm{S}$ with no dye added.

\section{AUTHOR INFORMATION}




\section{Corresponding Author}

*Address correspondence to xwdu@tju.edu.cn.

\section{Notes}

The authors declare no competing financial interest.

\section{ACKNOWLEDGMENTS}

This work was supported by the National Basic Research Program of China (2014CB931703), the Natural Science Foundation of China (Nos. 51171127, 51102176, and 51271129). Research carried out in part at the Center for Functional Nanomaterials, Brookhaven National Laboratory was supported by the U.S. Department of Energy, Office of Basic Energy Sciences, under Contract No. DE-SCo012704.

\section{REFERENCES}

(1) Cheng, C.; Fan, H. J. Branched Nanowires: Synthesis and Energy Applications. Nano Today 2012, 7, $327-343$.

(2) Bierman, M. J.; Jin, S. Potential Applications of Hierarchical Branching Nanowires in Solar Energy Conversion. Energy Environ. Sci. 2009, 2, 1050-1059.

(3) Shao, F.; Sun, J.; Gao, L.; Yang, S.; Luo, J. Forest-like $\mathrm{TiO}_{2}$ Hierarchical Structures for Efficient Dye-Sensitized Solar Cells. J. Mater. Chem. 2012, 22, 6824-6830.

(4) Sun, K.; Jing, Y.; Li, C.; Zhang, X.; Aguinaldo, R.; Kargar, A.; Madsen, K.; Banu, K.; Zhou, Y.; Bando, Y.; Liu, Z.; Wang, D. 3D Branched Nanowire Heterojunction Photoelectrodes for High-Efficiency Solar Water Splitting and $\mathrm{H}_{2}$ generation. Nanoscale 2012, $4,1515-1521$

(5) Sun, Y.; Zhao, Q.; Gao, J.; Ye, Y.; Wang, W.; Zhu, R.; Xu, J.; Chen, L.; Yang, J.; Dai, L.; Liao, Z.-M.; Yu, D. In Situ Growth, Structure Characterization, and Enhanced Photocatalysis of High-Quality, Single-Crystalline ZnTe/ZnO Branched Nanoheterostructures. Nanoscale 2011, 3, 4418-4426.

(6) Kim, H.; Seol, M.; Lee, J.; Yong, K. Highly Efficient Photoelectrochemical Hydrogen Generation Using Hierarchical ZnO/WO Nanowires Cosensitized with CdSe/CdS. J. Phys. Chem. C 2011, 115, 25429-25436.

(7) Zhou, W.; Cheng, C.; Liu, J.; Tay, Y. Y.; Jiang, J.; Jia, X.; Zhang, J.; Gong, H.; Hng, H. H.; Yu, T.; Fan, H. J. Epitaxial Growth of Branched alpha- $\mathrm{Fe}_{2} \mathrm{O}_{3} / \mathrm{SnO}_{2}$ Nano-Heterostructures with Improved Lithium-Ion Battery Performance. Adv. Funct. Mater. 2011, 21, 2439-2445.

(8) Liu, J.; Cheng, C.; Zhou, W.; Li, H.; Fan, H. J. Ultrathin Nickel Hydroxidenitrate Nanoflakes Branched on Nanowire Arrays for High-Rate Pseudocapacitive Energy Storage. Chem. Commun. 2011, 47, 3436-3438.

(9) Mai, L.-Q.; Yang, F.; Zhao, Y.-L.; Xu, X.; Xu, L.; Luo, Y.-Z. Hierarchical $\mathrm{MnMoO}_{4} / \mathrm{CoMoO}_{4}$ Heterostructured Nanowires with Enhanced Supercapacitor Performance. Nat. Commun. 2011, 2, 381.

(10) Ye, B. U.; Kim, B. J.; Park, J.; Jeong, H. Y.; Park, J. Y.; Kim, J. K.; Hur, J.-H.; Kim, M. H.; Lee, J.-L.; Baik, J. M. ThreeDimensional Branched Nanowire Heterostructures as Efficient Light-Extraction Layer in Light-Emitting Diodes. Adv. Funct. Mater. 2014, 24, 3384-3391.

(11) Wang, D.; Qian, F.; Yang, C.; Zhong, Z. H.; Lieber, C. M. Rational Growth of Branched and Hyperbranched Nanowire Structures. Nano Lett. 2004, 4, 871-874.

(12) Dick, K. A.; Deppert, K.; Larsson, M. W.; Martensson, T.; Seifert, W.; Wallenberg, L. R.; Samuelson, L. Synthesis of Branched 'Nanotrees' by Controlled Seeding of Multiple Branching Events. Nat. Mater. 2004, 3, 380-384.

(13) Dong, A.; Tang, R.; Buhro, W. E. Solution-Based Growth and Structural Characterization of Homo- and Heterobranched Semiconductor Nanowires. J. Am. Chem. Soc. 2007, 129, 12254-12262.

(14) Guo, Z.; Chen, X.; Li, J.; Liu, J.-H.; Huang, X.-J. ZnO/CuO Hetero-Hierarchical Nanotrees Array: Hydrothermal Preparation and Self-Cleaning Properties. Langmuir 2011, 27, 6193-6200.

(15) Xia, X.; Tu, J.; Zhang, Y.; Wang, X.; Gu, C.; Zhao, X.-B.; Fan, H. J. High-Quality Metal Oxide Core/Shell Nanowire Arrays on Conductive Substrates for Electrochemical Energy Storage. ACS Nano 2012, 6, 5531-5538.

(16) Chen, X.-Y.; Ling, T.; Du, X.-W. Low-Temperature Synthesis of ZnO/CdS Hierarchical Nanostructure for Photovoltaic Application. Nanoscale 2012, 4, 5602-5607.

(17) Zhu, J.; Peng, H.; Marshall, A. F.; Barnett, D. M.; Nix, W. D.; Cui, Y. Formation of Chiral Branched Nanowires by the Eshelby Twist. Nat. Nanotechnol. 2008, 3, 477-481.

(18) Yang, X.; Zhuang, J.; Li, X.; Chen, D.; Ouyang, G.; Mao, Z.; Han, Y.; He, Z.; Liang, C.; Wu, M.; Yu, J. C. Hierarchically Nanostructured Rutile Arrays. Acid Vapor Oxidation Growth and Tunable Morphologies. ACS Nano 2009, 3, $1212-1218$.

(19) Lao, J. Y.; Wen, J. G.; Ren, Z. F. Hierarchical ZnO Nanostructures. Nano Lett. 2002, 2, 1287-1291.

(20) Cho, K.-S.; Talapin, D. V.; Gaschler, W.; Murray, C. B. Designing PbSe Nanowires and Nanorings through Oriented Attachment of Nanoparticles. J. Am. Chem. Soc. 2005, 127, 7140-7147.

(21) Cheng, C.; Liu, B.; Yang, H.; Zhou, W.; Sun, L.; Chen, R.; Yu, S. F.; Zhang, J.; Gong, H.; Sun, H.; Fan, H. J. Hierarchical Assembly of $\mathrm{ZnO}$ Nanostructures on $\mathrm{SnO}_{2}$ Backbone Nanowires: Low-Temperature Hydrothermal Preparation and Optical Properties. ACS Nano 2009, 3, 3069-3076.

(22) Ma, C.; Moore, D.; Li, J.; Wang, Z. L. Nanobelts, Nanocombs, and Nanowindmills of Wurtzite ZnS. Adv. Mater. 2003, 15, 228231

(23) Zhuang, Z. B.; Peng, Q.; Li, Y. D. Controlled Synthesis of Semiconductor Nanostructures in the Liquid Phase. Chem. Soc. Rev. 2011, $40,5492-5513$.

(24) Wang, Q.; Xu, G.; Han, G. Synthesis and Characterization of Large-Scale Hierarchical Dendrites of Single-Crystal CdS. Cryst. Growth Des. 2006, 6, 1776-1780. 
(25) Grebinski, J. W.; Hull, K. L.; Zhang, J.; Kosel, T. H.; Kuno, M. Solution-Based Straight and Branched CdSe Nanowires. Chem. Mater. 2004, 16, 5260-5272.

(26) Li, D. S.; Soberanis, F.; Fu, J.; Hou, W. T.; Wu, J. Z.; Kisailus, D. Growth Mechanism of Highly Branched Titanium Dioxide Nanowires via Oriented Attachment. Cryst. Growth Des. 2013, 13, 422-428.

(27) Cademartiri, L.; Ozin, G. A. Ultrathin Nanowires - A Materials Chemistry Perspective. Adv. Mater. 2009, 21, $1013-1020$.

(28) Nakajima, T.; Shinoda, K.; Tsuchiya, T. UV-Assisted Nucleation and Growth of Oxide Films from Chemical Solutions. Chem. Soc. Rev. 2014, 43, 2027-2041.

(29) Kokai, F.; Nozaki, I.; Okada, T.; Koshio, A.; Kuzumaki, T. Efficient Growth of Multi-Walled Carbon Nanotubes by ContinuousWave Laser Vaporization of Graphite Containing B $\mathrm{B}_{4}$ C. Carbon 2011, 49, 1173-1181.

(30) Kingston, C. T.; Jakubek, Z. J.; Denommee, S.; Simard, B. Efficient Laser Synthesis of Single-Walled Carbon Nanotubes through Laser Heating of the Condensing Vaporization Plume. Carbon 2004, 42, 1657-1664.

(31) Sugiyama, T.; Yuyama, K.; Masuhara, H. Laser Trapping Chemistry: From Polymer Assembly to Amino Acid Crystallization. Acc. Chem. Res. 2012, 45, 1946-1954.

(32) Banerjee, R.; Jayakrishnan, R.; Ayyub, P. Effect of the Size-Induced Structural Transformation on the Band Gap in CdS Nanoparticles. J. Phys. - Condens. Mat. 2000, 12, 10647-10654.

(33) Nag, A.; Hazarika, A.; Shanavas, K. V.; Sharma, S. M.; Dasgupta, I.; Sarma, D. D. Crystal Structure Engineering by Fine-Tuning the Surface Energy: The Case of CdE (E = S/Se) Nanocrystals. J. Phys. Chem. Lett. 2011, 2, 706-712.

(34) Jiang, F.; Liu, J.; Li, Y.; Fan, L.; Ding, Y.; Li, Y. Ultralong CdTe Nanowires: Catalyst-Free Synthesis and High-Yield Transformation into Core-Shell Heterostructures. Adv. Funct. Mater. 2012, 22, 2402-2411.

(35) Shanavas, K. V.; Sharma, S. M.; Dasgupta, I.; Nag, A.; Hazarika, A.; Sarma, D. D. First-Principles Study of the Effect of Organic Ligands on the Crystal Structure of CdS Nanoparticles. J. Phys. Chem. C 2012, 116, 6507-6511. 